\title{
La prothèse plastique faciale
}

\section{RÉSUMÉ}

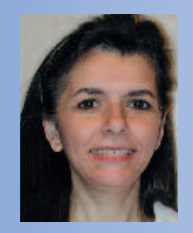

Ilham EL ACHHAB

Prothésiste maxillo-faciale et épithésiste,

Département

de cancérologie cervico-faciale,

odontologie,

Institut Gustave Roussy,

39 rue Camille Desmoulins

94800 Villejuif.
La prothése plastique faciale reste toujours d'actualité, elle est une alternative à la reconstruction, malgré les importants progrés de celle-ci. La demande des patients pour ce type d'épithèse est sans cesse croissante avec le développement des nouveau matériaux et leurs technologies, qui va redonner au patient une vie relationnelle, sociale et esthétique.

Dr J.-P. MARGaINAUd Chef du service d'odontologie de l'Institut Gustave Roussy, 94800 Villejuif
AOS 2012;260:327-339

DOI: $10.1051 /$ aos/2012404

(C) EDP Sciences
- fixation
- épithèse
- couleur
- fonctionalité
esthétique 


\section{Introduction}

$>$

La prothèse plastique faciale a pour objectif de restaurer des malformations et des mutilations faciales qui ne peuvent être reconstruites par chirurgie.

La prothèse maxillo-faciale est une alternative ou un complément à la chirurgie reconstructrice, malgré les progrès de celle-ci notamment dans la reconstruction de lambeaux pédiculés, myocutanés ou ostéomyocutanés et des lambeaux libres [2,12-14].
Le processus conduisant à la mise en place d'une épithèse ne se résume pas à la simple confection de sa forme, bien que cela soit le point central.

Le mode de fixation de l'épithèse est un prérequis avant l'étape de confection car il détermine une partie de sa forme et de son modelage.

Un travail de maquillage et de personnalisation (faux cil, sourcil, moustache, chevelure et barbe) clôture le travail.

\section{Détermination du mode de fixation}

La fixation est adaptée et a plusieurs exigences:

- la stabilité afin que l'épithèse ne se déplace pas, ni ne tombe ;

- le confort, afin d'être tolérée ;

- participer au mouvement de la mimique ;
- être invisible.

Le prothésiste maxillo-facial doit adapter chaque solution à chaque cas particulier.

Il doit « jouer » d'ingéniosité en fonction du terrain, de l'état de cicatrisation du patient.

\section{Différents types de fixation}

\section{Implants}

Dans le cas de la mise en place d'un système implantaire, l'association des résines et des silicones est devenue systématique.

Ces épithèses ont différents systèmes d'ancrage. Ils sont positionnés sur le visage du patient avec des implants endo-osseux du type Nobel Biocare ${ }^{\circledR}$ (Bränemark $\left.{ }^{\circledR}\right)$, Straumann $\left.{ }^{\circledR}\right)[2$, 8-13].

Cette technique est de plus en plus utilisée.
- Ses avantages sont :

- la facilité de mise en œuvre ;

- la bonne rétention.

- Son inconvénient majeur est le manque de place dans certain cas.

\section{Rétention anatomique}

Elle consiste à utiliser les reliefs et les dépressions naturelles comme un moyen de rétention. 
- Son avantage principal est qu'elle ne nécessite aucun autre moyen de fixation puisque la cavité est auto-rétentive.

- Ses inconvénients sont :

- ses limites puisque certaines cavités n'ont pas de rétention suffisante ;

- ses difficultés d'usage lorsque le patient ne peut insérer et désinsérer son épithèse facilement.

\section{Rétention mécanique}

Elle peut être assurée par les adhésifs, les colles ou les rubans adhésifs à double face.

\section{> Adhésifs}

Ils se présentent sous la forme d'un liquide qui est appliqué au pinceau sur l'épithèse et qui permet qu'elle soit collée à la peau.

- Leur avantage principal est de permettre un bon maintien de l'épithèse.

\section{- Mais leurs inconvénients sont nombreux :}

- irritations locales avec le temps, causées par le contact de la colle sur la peau et la succession des collages-décollages ;

- salissures dues à l'accumulation de la colle, notamment dans le cas où le patient est peu méticuleux. Ces salissures peuvent provoquer une surélévation de la colle ;

- inefficacité notamment dans les pays chauds car la colle ne résiste pas à la chaleur.

\section{> Rubans adhésifs}

Ils sont composés d'un support en polyester dont les deux faces sont enduites d'un adhésif.

- Leur avantage est leur facilité d'utilisation, de stockage et de conservation.

- Leur inconvénient est d'entraîner une certaine surélévation par rapport à la peau, ce qui peut rendre les bords de l'épithèse plus visible.

\section{Utilisation de paire de lunettes}

C'est un élément de choix dans la stabilisation de l'épithèse. La paire de lunette peut être solidaire à la prothèse ou être fixée par un dispositif amovible. La monture doit être suffisamment épaisse pour dissimuler l'attachement et les bords de la prothèse.

Des accessoires comme les cils, sourcils, moustache, cheveux sont aussi utilisés pour masquer et dissimuler certains bords de l'épithèse. Cela aide à son intégration par le patient.

\section{Création de l'épithèse}

Pour les épithèses, différents matériaux vont être associés [1, 2].

Les silicones trouvent de plus en plus d'indications, parce qu'ils ne provoquent pas de réactions inflammatoires. Ils peuvent se colorer et s'adapter aux formes les plus complexes grâce à leur élasticité et à leur facilité de mise en œuvre.
Les résines acryliques durent davantage dans le temps et peuvent être une bonne alternative lorsqu'un contexte particulier l'exige.

L'épithèse se crée en trois étapes :

- la prise de l'empreinte,

- la sculpture d'une maquette en cire,

- et la confection de l'épithèse en silicone. 


\section{Première étape : \\ prise de l'empreinte}

Les empreintes sont réalisées, soit par un alginate recouvert de bande plâtrée pour rigidifier l'ensemble, soit avec un élastomère, qui est beaucoup moins fragile qu'un alginate, surtout si la cavité est très rétentrice ou s'il y a des implants.

Les parties trop rétentrices sont comblées avec une gaze vaselinée, pour éviter que les matériaux utilisés ne fusent trop loin dans la cavité.

Dans le cas d'une épithèse nasale, le front, le bord inférieur de la lèvre supérieure (ceci permet au patient de respirer), la perte de substance, les yeux et joues sont moulés.

De cette empreinte, le modèle en plâtre dur est réalisé.

Lors de l'examen clinique du patient, le prothésiste maxillo-facial recherche les points d'appuis fermes et les sillons où il sera possible de dissimuler les bords.

Le tracé des limites de l'épithèse se fera sur le modèle en plâtre.

Les mêmes étapes ayant guidé la confection d'une épithèse nasales sont suivies pour une épithèse oculo-palpébrale et auriculaire.

De la même manière le mode de rétention doit être préalablement déterminé avant la confection de celle-ci.

Encore une fois la solution optimale à privilégier est la fixation implanto-portée. Des pièces de transfert sont positionnées sur les implants du patient.

Le moulage est pris sur la totalité de la face.

Sur ces transferts, on repositionne les répliques d'implants. Enfin, un modèle en plâtre est coulé à partir de cette empreinte.

\section{Deuxième étape : sculpture d'une maquette en cire}

Sur le tracé en plâtre, une première maquette en cire est confectionnée.

Elle est reportée et retravaillée sur le patient afin de compléter et d'affiner les contours, la forme et la dimension.

Ce premier essayage permet d'apprécier si la prothèse est en harmonie avec l'ensemble du visage et de réajuster la maquette en cire sur le modèle de travail en fonction du tracé initial.

Dans le cas d'une épithèse sur implant, sur les répliques d'implants, les aimants seront positionnés sur une petite plaque en résine sur laquelle la sculpture sera effectuée, en cas de choix d'attachement magnétique sachant que d'autres systèmes existent.

Le prothésiste maxillo-facial essaye la plaque aimantée sur le patient, repère les zones de rétention, le tracé et la limite de la future prothèse, en utilisant tous les reliefs naturels pour dissimuler la zone de jonction.

\section{Troisième étape : confection de l'épithèse en silicone [4]}

Un moufle à prothèse dentaire est utilisé. Il se présente en deux parties (la partie et la contrepartie) qui se séparent en deux. Le plâtre de classe II, est utilisé pour la mise en moufle. Celui-ci est coulé dans la première partie.

L'épithèse de la face interne est mis dans ce plâtre semi-liquide. Les bords libres sont dégagés. Après durcissement, cette première partie est isolée dans l'eau savonneuse, la contrepartie de la face externe de l'épithèse est coulée. Le moufle est ébouillanté afin de retirer toute la cire, ainsi on dispose pour chacun des 
moufles d'une partie et d'une contrepartie représentant chacune une moitié de la pièce, vernissage à chaud.

Les différentes teintes des régions sont reportées sur le moufle, les ombres, les veinules et les grains de beauté, ainsi que la teinte de base. La contrepartie est fermée sur la partie. Il est mis sous bride, la polymérisation se fait à froid et sous vide.
Pour une épithèse oculo-palpébrale, le globe oculaire artificiel impose certaines précautions. Un axe sur l'iris sera positionné afin que l'iris ne se déplace pas lors de l'ébouillantage. La teinte de base est prise sur le patient, le maquillage en surface est effectué. Afin que celle-ci passe inaperçue et soit la plus naturelle possible, des cils et des sourcils sont implantés $[8,9]$.

\section{Cas particuliers en fonction des régions}

\section{Épithèse oculo-palpébrale}

Le rebord sourcilier permet de dissimuler le bord supérieur de la prothèse.

Les bords inférieurs externes et internes sont masqués par la monture de la lunette.

Le globe oculaire est mis sur le patient, il est positionné par rapport au globe oculaire du côté sain et placé dans tous les sens de l'espace. Le prothésiste maxillo-facial tient compte de la proéminence du globe au-dessus, derrière le patient, dans le sens vertical et transversal.

A l'aide d'une réglette ophtalmique, on mesure la distance de la pupille par rapport à la ligne médiane du visage. Elle doit être la même que le côté opposé.

En se plaçant devant le patient, on vérifie que les deux globes sont au même niveau sur une ligne horizontale.

La sculpture de l'ensemble de la prothèse peut être commencée.

Les replis et les sillons sont reproduits. L'ouverture de la fente palpébrale est déterminée par rapport au côté sain.

Plusieurs essayages sont nécessaires pour régler l'ouverture de la paupière supérieure.
Le maintien de la prothèse oculo-palpébrale se fera grâce aux implants endo-osseux.

\section{Épithèse auriculaire}

Le travail de sculpture est difficile car il est effectué par rapport au modèle en plâtre de l'oreille saine. La sculpture est commencée par des formes approximatives puis progressivement les zones les plus complexes et plus fines sont abordées.

La prothèse doit être légère et mince.

La face interne est évidée derrière les reliefs de la face externe de l'hélix et de l'anthélix. Au cours de l'essayage de la maquette sur implant, le prothésiste maxillo-facial vérifie que la distance entre le bord supérieur de I'hélix et la région temporale soit identique des deux côtés. Celle-ci est plus visible de dos que de face.

Le grain de peau sera apporté sur l'épithèse. La teinte de base du patient présente de grandes variétés en fonction des émotions, de la température intérieure et extérieure. Le prothésiste maxillo-facial doit concilier ces contradictions tout en évitant une teinte trop uniforme. 


\section{Épithèse nasale}

Au niveau des ailes et de la columelle, la ligne de raccordement de la prothèse est arrondie, de façon à former un angle rentrant. Latéralement, les bords sont effilés pour atteindre la partie supérieure du sillon nasogénien.

En haut de la prothèse, des extensions sont prévues sur lesquelles la monture de lunette va prendre appui. Cette maquette est creuse pour la rendre légère.

Le patient est revu pour le deuxième essayage, l'ajustage de la monture à la lunette est préparé. L'extrémité supérieure de la maquette est rectifiée de façon à dissimuler complètement derrière le pont de la monture. Les extensions destinées à supporter les plaquettes sont retouchées pour s'adapter à la prothèse, tout en restant aussi peu visibles que possible. Le bord de la prothèse est également retouché pour s'effacer derrière la monture.

Sur le modèle en plâtre, les formes qui avaient été ébauchées au cours de l'essayage sont retouchées. Les sculptures du relief de la peau sont effectuées en harmonie avec le reste du visage.

À la fin de la réalisation de cette maquette en cire, une première teinte, dite de base, est ébauchée en respectant les unités et les sousunités esthétiques qui suivent les zones d'ombre et de lumière du visage.

Si le moyen de rétention de l'épithèse est la paire de lunettes, celle-ci viendra s'appuyer sur la partie supérieure, pour stabiliser la fixation.

\section{Maquillage}

Le maquillage des épithèses est une autre spécificité. L'art du maquillage demande à être
L'avantage de ce procédé est de retirer les lunettes sans enlever la prothèse nasale.

Dans le cas où une communication buccosinusienne existe et que le patient porte une prothèse obturatrice, le prothésiste maxillofacial va essayer d'utiliser cette prothèse obturatrice pour fixer un système magnétique afin que l'épithèse nasale vienne s'emboîter à l'obturateur. Cette technique est avantageuse lorsque le patient ne peut être implanté suite à sa radiothérapie. Les amputations partielles sont plus difficiles à appareiller que la perte totale de l'organe. Les bords de l'épithèse sont dissimulés dans un pli naturel. Notamment dans le cas des prothèses nasales partielles et du pavillon de l'oreille particulièrement difficile, car ce sont des organes dont la teinte varie considérablement dans la même journée. Il est très difficile pour le prothésiste maxillo-facial de trouver une teinte qui s'harmonise avec la peau voisine.

Les bords de la perte de substance sont fragiles. Les dimensions de celles-ci peuvent se modifier. En effet, le résultat est le plus souvent décevant car les limites et les zones de transition entre prothèse et peau du patient sont toujours visibles.

L'ensemble de ces raisons, explique pourquoi une prothèse totale est préférée et un geste d'amputation complémentaire est programmé dans le cas où une restauration épithétique est définitivement actée par le patient et l'équipe soignante $[10,11]$.

maîtrisé par le prothésiste principalement lors de la réalisation d'épithèses. 
Il se fait grâce à une colle à froid additionnée à du cyclohexane (un diluant) et à des colorants. Sans cette colle à froid, le maquillage ne peut adhérer sur l'épithèse.

Le maquillage de celle-ci se fait avec une gestuelle adaptée à chaque patient. Pour que celui-ci ne fasse pas artificiel, les détails et les défauts sont reproduits au plus proche de I'identique (taches de rousseur, grains de beauté, vaisseaux sanguins, rides, ombres au niveau des ailes du nez...).

\section{La couleur de la peau varie en fonction de plusieurs facteurs}

- La teinte de base est constituée par des pigments de peau qui varient en fonction des races et des taches pigmentaires, dont la répartition est très variable [3]. Le maquillage nuancera des teintes allant du jaune au brun foncé.

- La couleur de la peau est influencée par la réflexion de la lumière sur la couche superficielle de l'épiderme. Celle-ci est translucide et sa surface est granuleuse. Elle permet la réflexion de la lumière. C'est la raison pour laquelle certaines prothèses faciales, qui paraissent s'intégrer parfaitement à la teinte du visage sous la lumière du jour, sont plus visibles à la lumière artificielle ou inversement.

Le prothésiste maxillo-facial attache une grande importance à la surface de la prothèse mais aussi à la coloration de celle-ci.

- La vascularisation superficielle apporte à la peau des variations de teinte. Les dilatations vasculaires peuvent apparaître sous forme de varicosités, notamment sur le nez et les pommettes. Elles sont reproduites sur la prothèse faciale en incorporant des veinules colorées dont la base est faite de rouge et de bleu.
- Le système pileux de l'homme brun doit être reproduit également. Lorsqu'il se rase, le bleuté devient prédominant sur les joues et le menton. La teinte bleu doit être reproduite.

Un maquillage d'épithèse nécessite trois à quatre heures de travail.

\section{Plusieurs éléments complexifient la réalisation du maquillage \\ $>$ Le temps}

Le maquillage ne peut se faire qu'en présence du patient. II doit donc rester positionné plusieurs heures de suite.

\section{> La température de la pièce}

Si le patient a chaud, il rougit, sa teinte se modifie et le maquillage est faussé. Les variations sont surtout visibles sur les joues, les ailes et la pointe du nez, la partie inférieure du front et le pavillon de l'oreille.

\section{> Les émotions et l'état de fatigue}

En cas de variations trop importantes, la teinte du bras du patient servira alors de référence.

\section{> Les peaux foncées}

Certains types de peau sont plus difficiles à reproduire, c'est le cas des peaux de type africain, car elles nécessitent un mélange de couleurs beaucoup plus subtiles que les peaux de type européen ou asiatique.

\section{$>$ Les yeux}

Pour les prothèses oculaires, la difficulté consiste à placer l'iris. La position doit être parfaite pour éviter les effets de strabisme et d'exophtalmie ou d'enophtalmie. 


\section{Cas cliniques}

Différentes étapes de confection d'une épithèse auriculaire collée

(fig. 1 à 7)

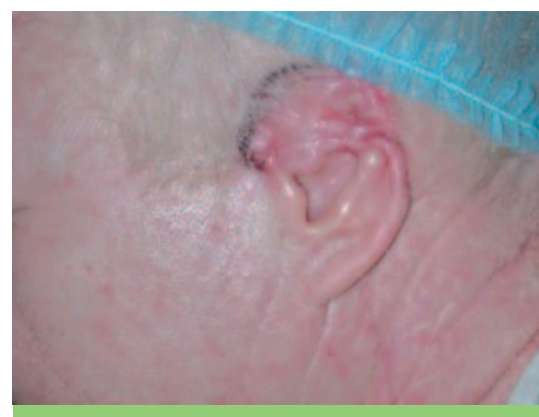

Fig. 1 Prise d'empreinte et traçage de la partie manquante.

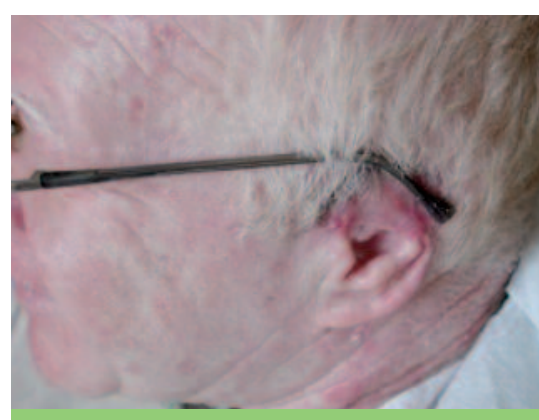

Fig. 2 Visualisation de la branche de lunette, sur la partie manquante où sera posée l'épithèse finie.

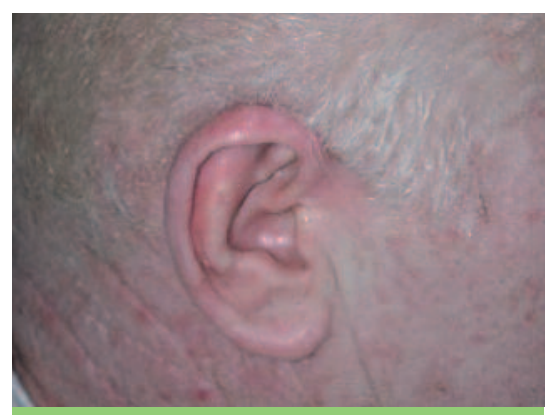

Fig. 3 Prise d'empreinte de l'oreille collatérale.
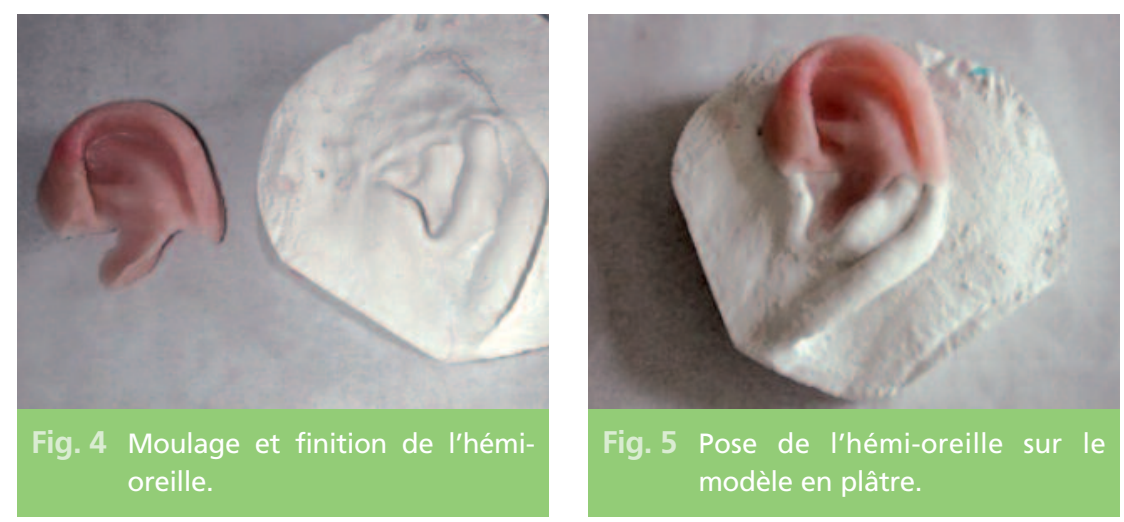

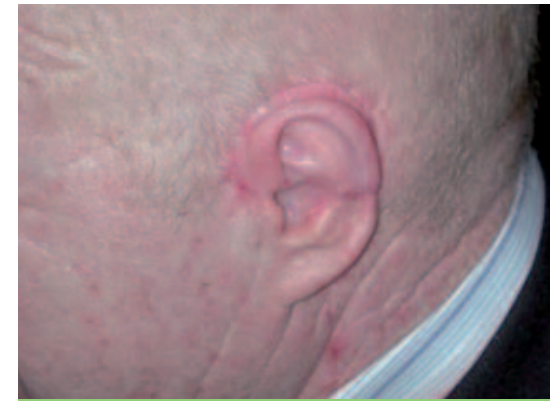

Fig. 6 Finition, maquillage et pose.

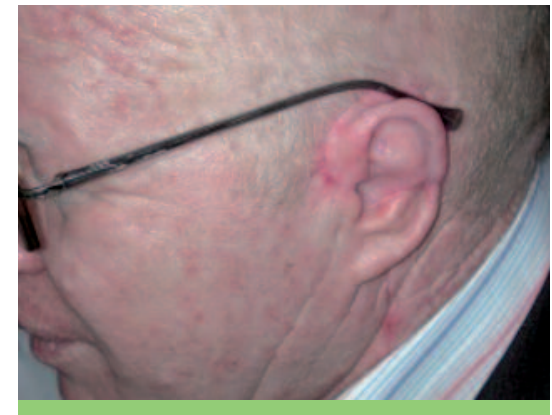

Fig. 7 Positionnement de la paire de lunettes sur l'épithèse collée. Celle-ci permettra le calage de l'épithèse. 
Différentes étapes de confection

d'une épithèse auriculaire aimantée

(fig. 8 à 22)

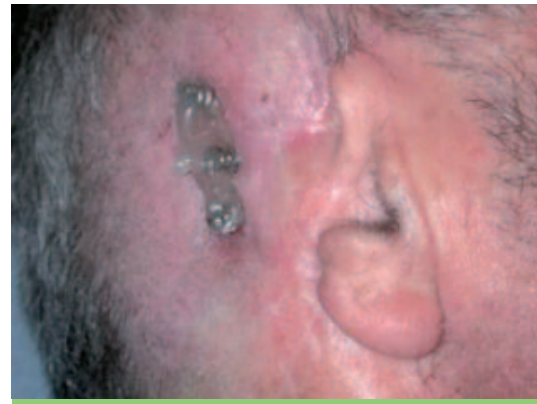

Fig. 8 Prothèse auriculaire sur implant.

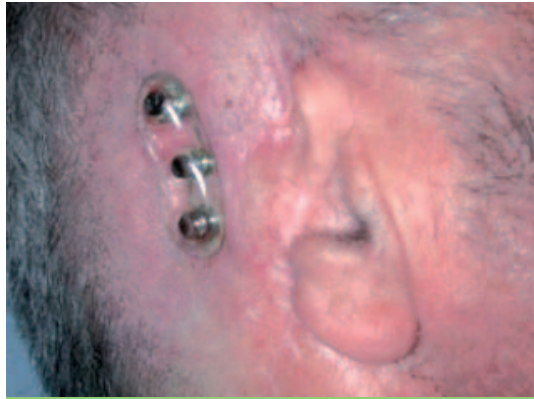

Fig. 9 Conformateur sur une base acrylique aimantée.

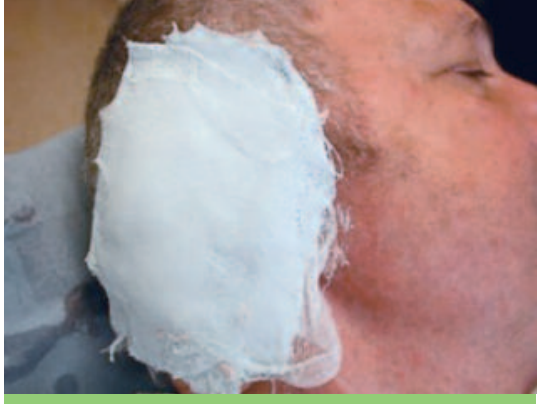

Fig. 10 Prise d'empreinte.
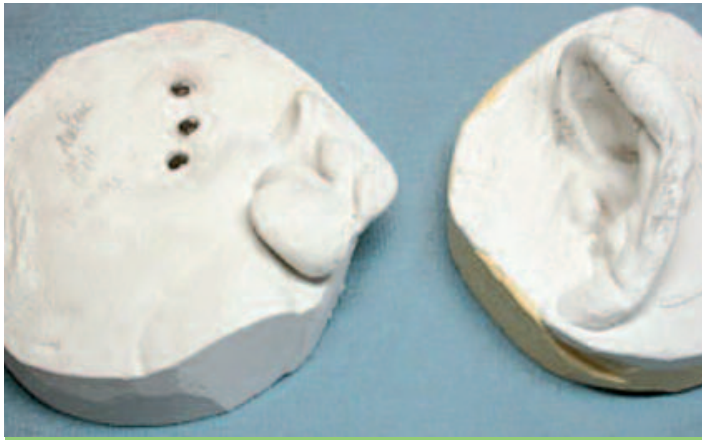

Fig. 11 Modèle en plâtre pour la confection de la maquette en cire.

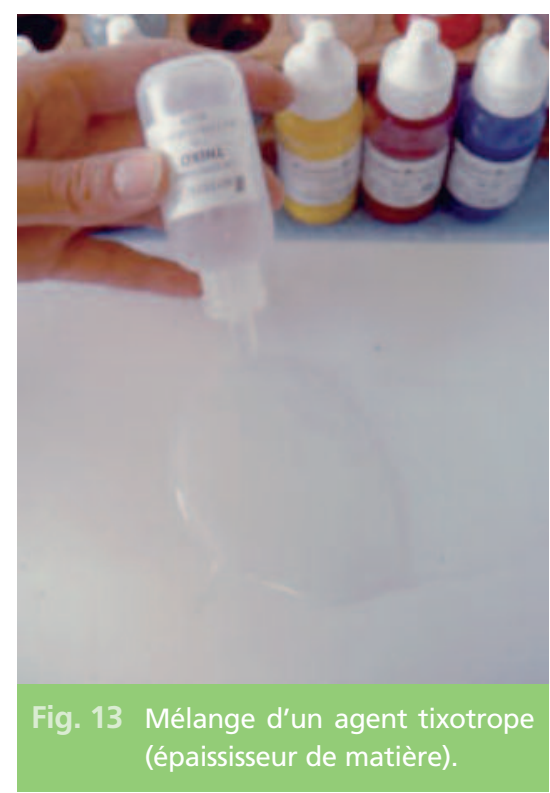

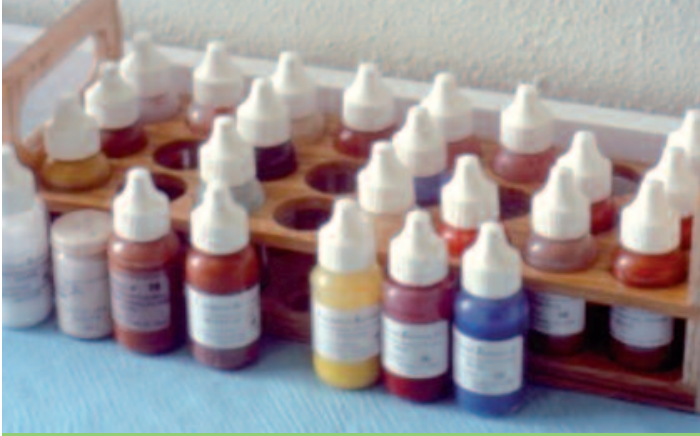

Fig. 12 Kit pour colorer un silicone.

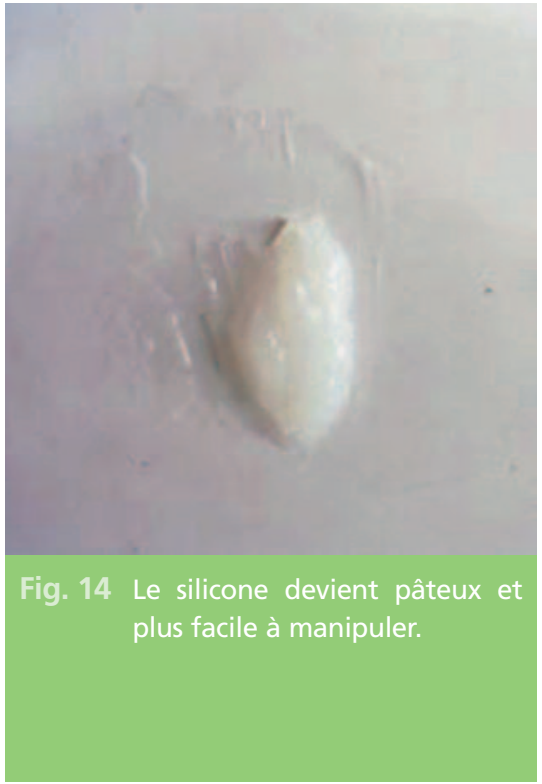



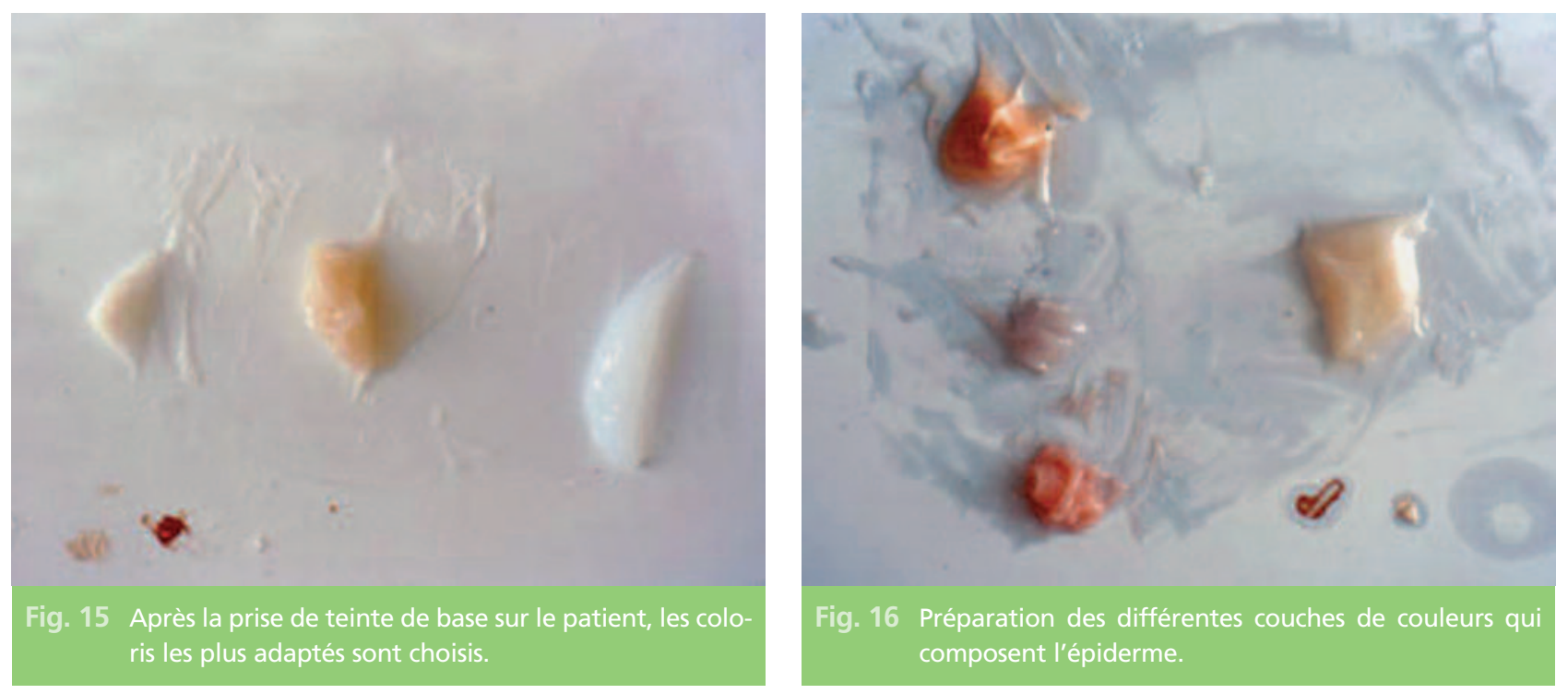

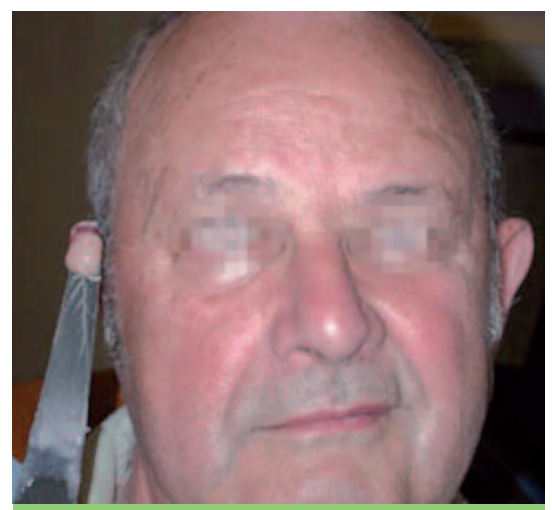

Fig. 17 Teinte de base du patient.

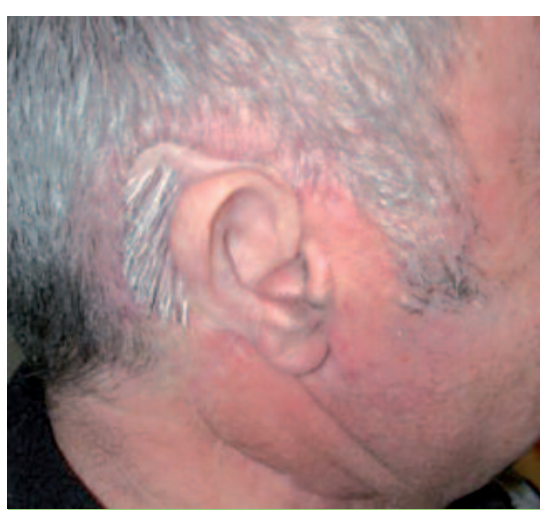

Fig. 20 Pose de la prothèse finie en silicone.

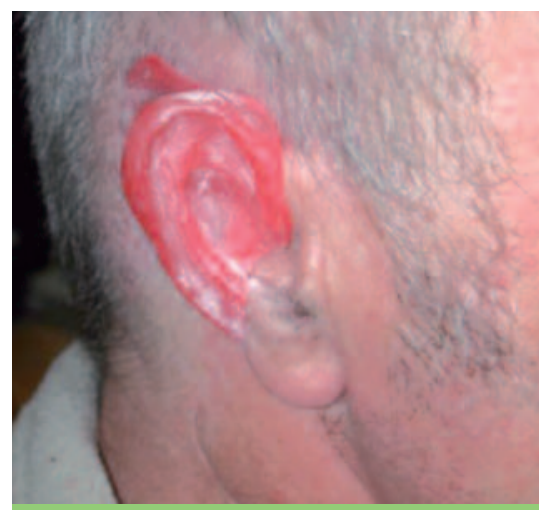

Fig. 18 Maquette en cire essayée sur le patient.

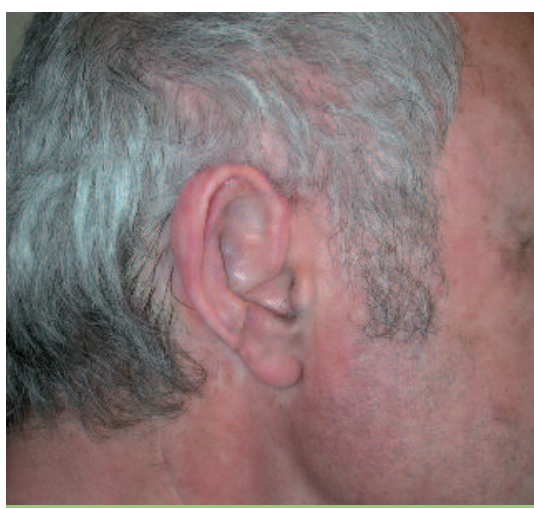

Fig. 21 Maquillage de l'épithèse en silicone. Personnalisation de celle-ci, des cheveux sont incrustés dans l'épithèse afin que celle-ci passe inaperçue.

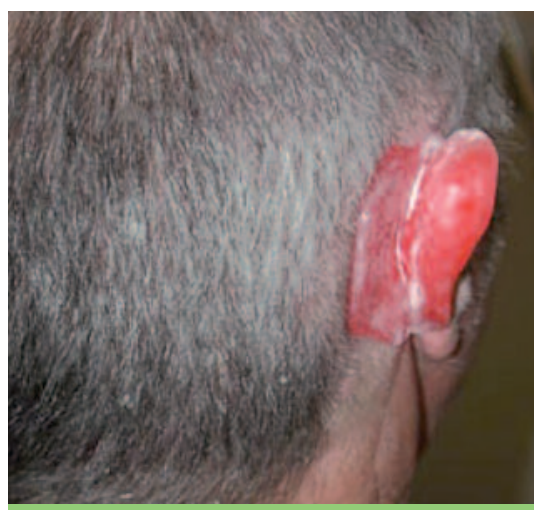

Fig. 19 Maquette en cire essayée sur le patient.

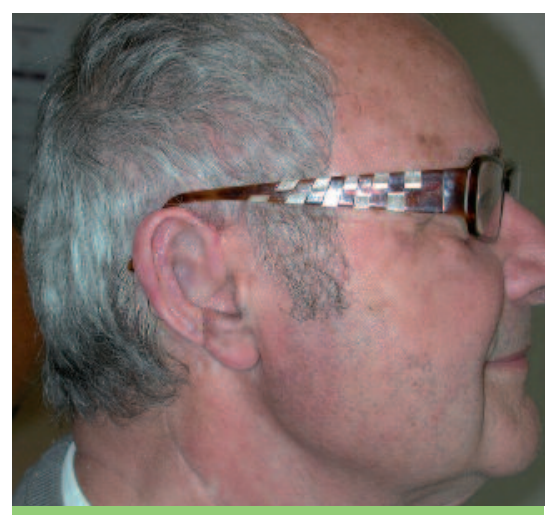

Fig. 22 Finition. 
Différentes étapes de confection d'une épithèse nasale (fig. 23 à 26)

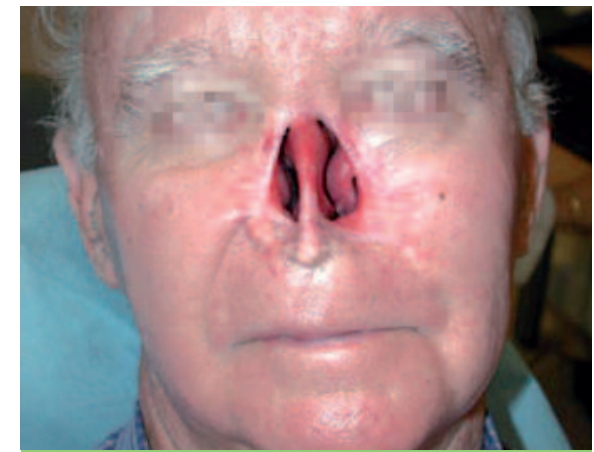

Fig. 23

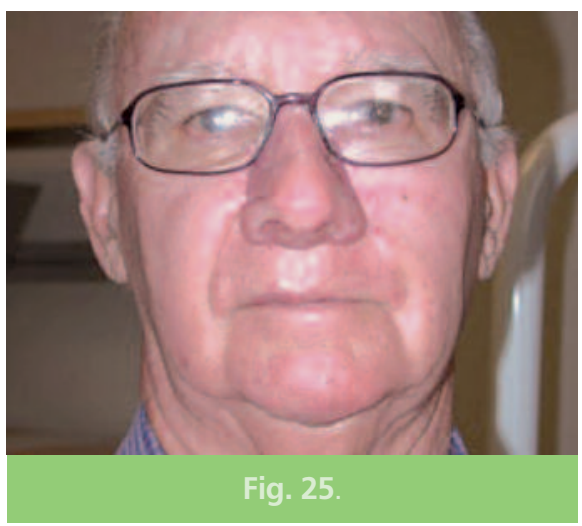

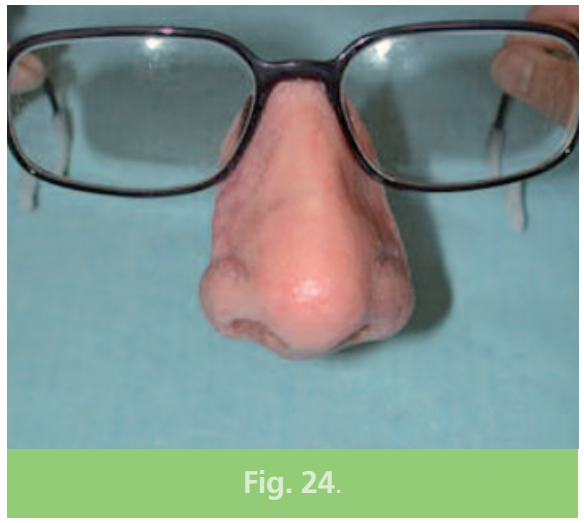

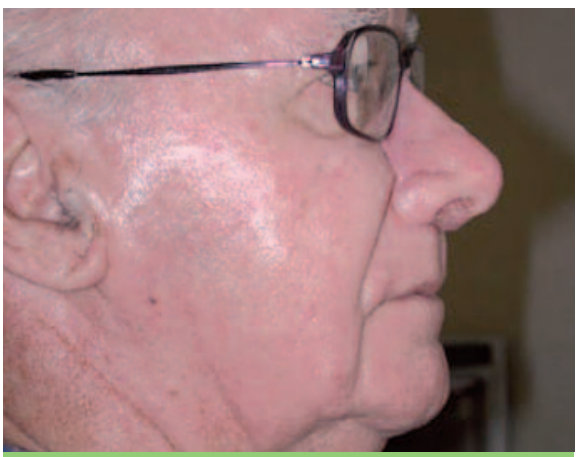

Fig. 26.

Différentes étapes de confection d'une épithèse oculo-palpébrale sur implant (fig. 27 à 29)

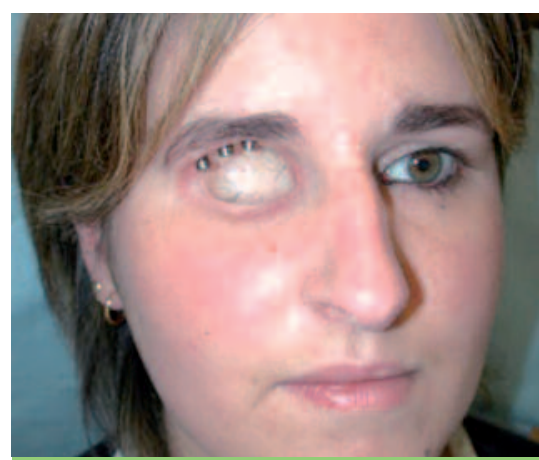

Fig. 27 Épithèse oculaire : la fixation se fera sur une base acrylique aimantée.

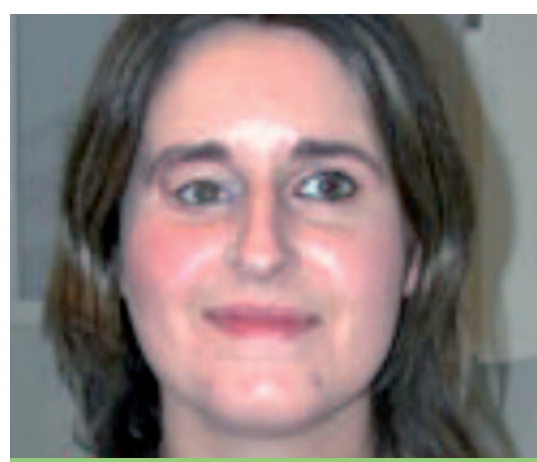

Fig. 28 Maquillage et pose.

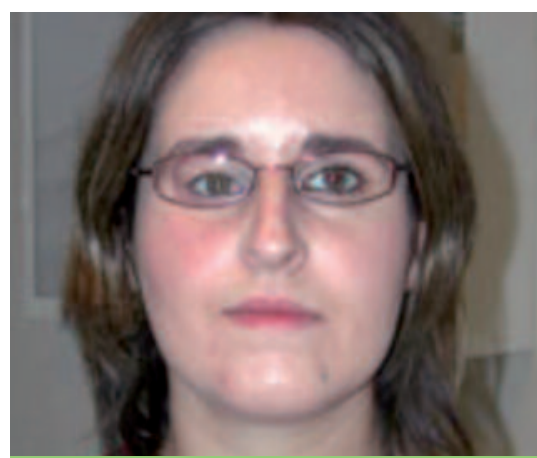

Fig. 29 Finition. 


\section{Conclusion}

Certains malades n'acceptent pas leurs infirmités. Le port d'une prothèse, même de conception parfaite, leur semble inutile car elle ne permet pas de restituer leur état antérieur. Le prothésiste maxillo-facial et I'odontologue se doivent d'être persuasifs en expliquant le bénéfice fonctionnel et esthétique, la phase d'adaptation nécessaire, et qu'il ne sera pas seul toute cette période, une aide psychologique sera apportée. Un patient ne portera sa prothèse que si elle correspond à un besoin fonctionnel, esthétique et psychologique [5-7]

Le prothésiste maxillo-facial doit souvent trouver de nouvelles possibilités, à imaginer de nouveaux montages. Ce travail se fait en étroite collaboration avec les odontologues et les chirurgiens. Grâce au progrès des matières en silicone, il est possible de réaliser des prothèses dont l'esthétique est supérieure à une reconstruction chirurgicale. Ces prothèses faciales passent inaperçues et s'intègrent bien au visage grâce à la texture et à la coloration de l'épithèse.
Le goût pour le beau occupe une place prépondérante dans ce travail, qu'il s'agisse du résultat final ou des matériaux manipulés.

Mais le plus gratifiant, c'est le lien social que la prothèse va pouvoir rendre à nouveau possible. La chirurgie ORL est souvent très dévastatrice sur le plan esthétique et laisse des visages profondément marqués qui rendent le contact à I'autre quasi impossible dans certains cas. Certains patients supportent difficilement l'amputation d'un organe de la face, ce qui les conduit à avoir une vie recluse, voir dégradante et moralement difficilement supportable pour eux et leurs proches. La prothèse va permettre aux patients de recouvrer l'estime de soi, de sortir à nouveau et d'oser montrer leur visage. Elle va également parfois leur offrir la possibilité de parler à nouveau et de s'alimenter. Ces prothèses conçues et façonnées pour eux rétablissent un sentiment de sécurité et de confiance qui permet aux patients de sourire à nouveau, au sens propre comme au figuré. Beaucoup de personnes sont porteuses de prothèse, sans que cela se voit ou se sache.

\section{Bibliographie}

1. Raes J.

Matériaux utilisées en prothèse maxillo-faciale. Rev Fr Réhabil Prothèse Maxillofac 1981;10:35-46.

2. Wolfaardt JF, Chandler HD, Smith BA. Mechanical properties of a new facial prosthetic materiel. J Prosthet Dent 1985;53(2):228-34.
3. Gary JJ, Smith CT. Pigments and their application in maxillofacial elastomers: a literature review. J Prosthet Dent 1998;80(2):204-8.

4. Soulet $\mathrm{H}$. Reconstruction prothétique de la peau à base de silicone. Thèse chir. dentaire.
Toulouse, 1973. AFM 731.

5. Saade AK, Caron J, Pomar P, Toulouse E, Poulain G.

Relation cabinet-laboratoire en prothése maxillo-faciale. Réalités Cliniques 2002;13(2):191-201.

6. Vigarios $\mathrm{E}$, Fontes-Carrere $\mathrm{M}$, Pomar P, Bach K. Psychologie et relation d'aide 
en prothèse maxillo-faciale. Encycl Méd Chir (Elsevier SAS, Paris), Stomatologie, 2004;22-066-B-53.

7. Vigarios $E$, Pomar $P$, Toulouse E, Fusaro S, Grhenassia C. Épithèses faciales. Encycl Méd Chir (Elsevier SAS, Paris), Stomatologie, 2006;22-066-B-56..

8. Prutthi G, Jain V, Sikka S. A novel method for retention of an orbital prosthesis in a case with continuous maxillary and orbital defect.
J India Prosthodont Soc 2010;10(2):132-6.

9. Pomar $\mathrm{P}$, Soulet $\mathrm{H}$. Mise en œuvre d'un élastomère de silicone dans la réalisation d'organes artificiels faciaux. Act Odonto Stomatol 1996;193:79-92.

10. Benoist M, Dichamp J, Poulain G.

Les problèmes esthétiques de restauration prothétique de la face en fonction de l'environnement. Act Odonto Stomatol 1984;38(148):619-30.

11. McKinstry RE. Fundamentals of facial prosthetics.

Arlington: $A B I$ Professional Publications, 1995.

12. McCraw JB, Dibbell DG. Experimental definition of independent myocutaneous vascular territories.

Plast Reconstr Surg 1977;60(2):212-20.

13. Thomas KF. Prosthetic rehabilitation. London: Quintessence Book Co., 1994.

14. Reisberg DJ, Habakuk SW. Use of a surgical positioner for bone-anchored facial prostheses. Int J Oral Maxillofac Implants 1997;12(3):376-9.

\section{SUMMARY}

\section{Facial prosthetic rehabilitation}

Ilham EL ACHHAB

\section{Keywords \\ - attachment \\ - epithesis \\ - color \\ - functional \\ - esthetic}

Facial prosthetic rehabilitation remains an important alternative to surgical reconstrection, despite the improvements in surgical techniques in recent years. New materials and technology have expanded prosthetic possibilities to improve esthetic and social quality of life. 